\title{
Community-associated methicillin-resistant Staphylococcus aureus in non-outbreak skin infections
}

\author{
Mariana Fávero Bonesso ${ }^{1,2}$, Silvio Alencar Marques ${ }^{3}$, Carlos Henrique Camargo ${ }^{1}$, \\ Carlos Magno Castelo Branco Fortaleza ${ }^{2}$, \\ Maria de Lourdes Ribeiro de Souza da Cunha ${ }^{1}$ \\ ${ }^{1}$ Departamento de Microbiologia e Imunologia, Instituto de Biociencias de Botucatu, \\ Universidade Estadual Paulista "Júlio de Mesquita Filho", Botucatu, SP, Brazil. \\ ${ }^{2}$ Departamento de Doenças Tropicais, Faculdade de Medicina de Botucatu, \\ Universidade Estadual Paulista "Júlio de Mesquita Filho", Botucatu, SP, Brazil. \\ ${ }^{3}$ Departamento de Dermatologia e Radiologia, Faculdade de Medicina de Botucatu, \\ Universidade Estadual Paulista "Júlio de Mesquita Filho", Botucatu, SP, Brazil.
}

Submitted: September 17, 2013; Approved: April 17, 2014.

\begin{abstract}
The aim of this study was to determine the prevalence of Staphylococcus aureus and risk factors for the acquisition of MRSA (Methicillin Resistant Staphylococcus aureus) as the main cause of skin and soft tissue infections. S. aureus were characterized for the presence of PVL, TSST-1 and mecA genes. SCCmec typing was carried out in mecA positive strains and PFGE was performed only in these strains. During the study period, 127 outpatients attending a dermatology clinical the Botucatu Medical School, a regional tertiary hospital in Botucatu, Sao Paulo, Brazil, were diagnosed with active skin infections. A total $66(56.9 \%)$ S. aureus strains were isolated. The methicillin resistance gene mecA was detected in seven (10.6\%) S. aureus strains. The SCCmec types detected in the seven mecA-positive $S$. aureus strains were type Ia in one, type II in three, and type IV in three. The PVL gene was detected in $10(15.1 \%)$ in sensitive strains. Pulsed field gel electrophoresis revealed non-clonal diversity among the isolates. The risk factors associated with MRSA acquisition in this study were previous ciprofloxacin use and working in a healthcare environment. The risk factors indicate plausible routes of CA-MRSA transmission among the subjects studied.
\end{abstract}

Key words: Staphylococcus aureus, resistance, skin infections, virulence, epidemiology.

\section{Introduction}

Staphylococcus aureus is a versatile pathogen associated with infections in both hospital and community setting (Miranda et al., 2007, Schuenck et al., 2009, D'Agata et al., 2009). Due to its widespread use, treatment with penicillin became ineffective due to the high rate of resistance; consequently, semi-synthetic penicillin, such as methicillin, was developed as the main alternative against penicillin resistant strains. However soon before its introduction, Methicillin-Resistant S. aureus (MRSA) strains emerged in hospital environments (Jevons 1961) and then in the community about 20 years later (Gosbell 2004).
The main methicillin resistance mechanism is the production of PBP2a, a transpeptidase involved in bacterial wall synthesis, codified by the mecA gene (Chambers 1997) and carried in a mobile element called the staphylococcal cassette chromosome mec ( $\mathrm{SCCmec).} \mathrm{SCCmec} \mathrm{is}$ classified according to the presence or absence of specific DNA regions (Turlej et al., 2011). So far, 11 main SCCmec types (I to XI, and subtypes) have been described (IWGSCC). Usually, SCCmec types I, II and III are found in hospital environments (Hospital-Associated Methicillin Resistant S. aureus) while types IV, V and VI are associated with community settings (Community-Associated Methicillin

Send correspondence to: M.F. Bonesso. Departamento de Microbiologia e Imunologia, Instituto de Biociencias de Botucatu, Universidade Estadual Paulista "Júlio de Mesquita Filho", Caixa Postal 510, 18618-970 Botucatu, SP, Brazil. E-mail: mfbonesso@hotmail.com. 
Resistant $S$. aureus). However, according to recent data, a change in the epidemiology of CA-MRSA has been reported (Cook and Brown 2010).

Community-associated methicillin-resistant S. aureus (CA-MRSA)cause a broad range of skin and soft tissue infections such as furuncles, boils, carbuncles (Yao et al., 2010), cellulitis, and necrotizing fasciitis (Miller et al., 2005) in patients without traditional risk factors (Campbell et al., 2004). CA-MRSA strains usually carry the Panton Valentine leukocidin (PVL) gene as the main virulence factor. PVL is a two-component pore-forming cytotoxin that acts on mitochondria and polymorphonuclear leucocytes wich indirectly leads to epithelial necrosis and invasiveness (Boyle-Vavra and Daum 2007). In addition to reports demonstrating the importance of PVL for MRSA infections (David et al., 2008; Goering et al., 2008; Camargo et al., 2013), methicillin-sensitive S. aureus (MSSA) strains have also been shown to carry the PVL gene and to be responsible for severe infections (D'Azevedo et al., 2009; Lee et al., 2010; Kreienbuehl et al., 2011).

The objectives of the present study were to determine the prevalence of MRSA from a population attending an outpatient dermatology clinic, to evaluate associated risk factors, and to determine the virulence profile of $S$. aureus strains.

\section{Material and Methods}

\section{Study design}

This was a prospective cross-sectional study involving a convenience sample of outpatients presenting skin and/or soft tissue infections. The patients were seen at an emergency and/or outpatient dermatology clinic of the Botucatu Medical School, between September 2008 and September 2009. The patients provided written informed consent to participate in this study. The researcher was trained to interview the patients using a questionnaire to collect the following data: age, gender, residence area (urban or rural), working in a healthcare environment, previous institutional admission in the last 12 months (hospital, clinics, prison, sports team facilities), diabetes diagnosis, medical procedure in the last 12 months (surgery, dialysis, catheterization, drainage), illicit drug intake, sexual orientation, length of infection, antibiotic usage, sports practice, work status and income. We defined working in healthcare environment (healthcare worker) as a professional who directly assists patients.

\section{Inclusion criteria}

The patients were screened by the dermatologist during a medical consultation. Patients presenting active infections at the time of consultation who met the inclusion criterion for infections were included in the study. Infections included in this criterion were diagnoses for: boils, cellulitis, erysipelas, abscesses, hidradenitis, folliculitis, diabetes-related infected foot, suppurative osteomyelitis, secondarily infected dermatitis, secondarily infected traumatic lesions, and bullous impetigo. We considered patients who were hospitalized and healthcare workers to be at risk.

\section{Collection and bacterial isolation}

A moistened (sterile saline) swab was used to collect the clinical material. The cultures were immediately sent to the laboratory and streaked onto Sheep Blood Agar and Mannitol Salt agar and incubated at $37^{\circ} \mathrm{C}$. Staphylococcus spp. colonies were identified based on Gram stain, catalase and coagulase tube production. The isolates were stored in nutrient broth with glycerol at $-70^{\circ} \mathrm{C}$.

\section{Detection of methicillin resistance and antimicrobial susceptibility testing}

The disk diffusion test was performed using oxacillin $(1 \mu \mathrm{g})$ and cefoxitin $(30 \mu \mathrm{g})$ disks as described by the Clinical Laboratory Standards Institute (CLSI 2009). Additionally, we used the mecA gene detection as the gold standard for evaluating MRSA. The plates were incubated at $35^{\circ} \mathrm{C}$. Susceptibility to penicillin $(10 \mathrm{U})$, erythromycin $(15 \mu \mathrm{g})$, clindamycin $(2 \mu \mathrm{g})$, sulfamethoxazole/trimethoprim $(125 \mu \mathrm{g} / 23.75 \mu \mathrm{g})$, gentamicin $(10 \mu \mathrm{g})$ and linezolid $(30 \mu \mathrm{g})$ was tested according to CLSI guidelines. The mecA gene was detected by polymerase chain reaction (PCR) (Murakami et al., 1991) after bacterial DNA extraction (Pereira et al., 2009), and a positive result defined MRSA. Reference strains were included in all reactions as positive (S. aureus ATCC 33591) and negative (S. aureus ATCC 25923) controls for PCR reactions.

\section{Characterization of $S$. aureus SCCmec}

SCCmec typing was performed for mecA positive isolates only, using the primers and multiplex PCR parameters described by Milheiriço et al. (2007). The following reference strains were used: COL for SCCmec type I; N315 for SCCmec type Ia; PER34 for SCCmec type II; AN546 for SCCmec type III; HU25 for SCCmec type IIIa, and MW2 for SCCmec type IV.

\section{PCR detection of lukF-PV and lukS-PV and TSST-1 genes}

The genes and parameters standardized by Lina et al. (1999) were used for detecting the PVL genes (lukF-PV and lukS-PV). The toxic shock syndrome toxin 1 (TSST-1)gene was detected as proposed by Johnson (1991) and optimized by Cunha et al. (2006). S. aureus ATCC 49775 and S. aureus ATCC 29213 were used as positive and negative controls, respectively, in all reactions. 


\section{PFGE of MRSA isolates}

MRSA typing was performed by macrorestriction of genomic DNA of the strains, followed by PFGE according to McDougal et al. (2003). The gels were stained with the GelRed system (10,000x in water; Biotium, USA) for $1 \mathrm{~h}$ and photographed under UV transillumination. The BioNumerics software, version 6.1 (AppliedMaths, Belgium) was used for similarity analysis, calculation of the Dice correlation coefficient, and construction of a dendrogram by UPGMA (unweighted pair group method using arithmetic averages). Band position tolerance and optimization were set at $1.25 \%$ and $0.5 \%$, respectively. A similarity coefficient of $80 \%$ was chosen for the determination of clusters (McDougal et al., 2003). CA-MRSA representative clones (USA 500, USA400, USA 100, USA 800, USA 300, HU-25, EMRSA 15, JCSC 4469, OSPC, MR 108, NCTC 10422) were used for comparison in PFGE analysis.

\section{Statistical analysis of risk factors}

Risk factors were analyzed according to the demographic data collected (described in study design) from the population studied. The data were analyzed using the Epi Info ${ }^{\circledR}$ 3.5.2 (Centers for Disease Control and Prevention, Atlanta, USA) and SPSS 15.0 programs.

The results were submitted to univariate analysis. Dichotomous variables were analyzed by the chi-squared or Fisher's exact test. The Mann-Whitney test was applied to the analysis of numerical variables and to multivariate analysis using a logistic regression model. $\mathrm{p}$ values $<0.05$ and $>0.1$ were used for inclusion and exclusion of the variables in the model, respectively.

\section{Results}

A total of 127 swabs,one per patient,were obtained from patients seen in the emergency department and/or dermatology outpatient clinic during the study period, from which $S$. aureus was isolated in $66(52 \%)$. Coagulasenegative Staphylococcus strains were isolated in 42 (33\%) patients and the remaining $19(15 \%)$ patients presented negative culture for Staphylococcus. S. aureus were isolated from secondarily infected dermatitis/secondarily infected traumatic lesions (26 strains, 39.4\%), ingrown toenail infection (13 strains, 19.7\%), boils (8 strains, 12.1\%), bullous impetigo (6 strains, 9.1\%), diabetes-related infected feet (4 strains, 6.1\%) cellulitis, osteomyelitis (3 strains, $4.5 \%$, each), folliculitis, erysipelas and necrotic tissue (1 strain, $1.5 \%$, each).

\section{Identification of MRSA by the disk diffusion method and mecA gene detection}

Nine (13.6\%) S. aureus strains were resistant to oxacillin and eight $(12.1 \%)$ were resistant to cefoxitin. The mecA gene was detected in seven (10.6\%) isolates. Of these, six $(85.7 \%)$ were resistant to oxacillin and six
$(85.7 \%)$ to cefoxitin. On the other hand, among the mecA negative strains, 3 presented oxacillin resistance and 2 presented cefoxitin resistance.

\section{SCCmec typing of $S$. aureus and susceptibility profile of MRSA}

Among the seven $S$. aureus strains carrying the mecA gene, one (14.2\%) was classified as type Ia, three (42.9\%) as type II, and three (42.9\%) as type IV. S. aureus carrying SCCmec type Ia was only resistant to penicillin. The three strains carrying type II were resistant to cefoxitin, clindamycin, erythromycin, oxacillin, and penicillin. The three SCCmec type-IV isolates were resistant to cefoxitin, oxacillin and penicillin, but only one strain each (33.3\%) was resistant to clindamycin and erythromycin.

\section{Detection of the lukS-PV/lukF-PV genes and TSST-1 gene in S. aureus}

The TSST-1 gene was not detected in any of the strains studied. The lukSF-PV gene was detected in 10 $(15.1 \%)$ strains, only in methicillin-sensitive strains. Among the 10 S. aureus strains carrying the PVL genes, six $(60 \%)$ were recovered from furuncles, two $(20 \%)$ from secondary infections, and two (20\%) from impetigo.

\section{PFGE of MRSA}

Four isolates containing SCCmec II presented the same pulsotype in PFGE, including a bloodstream infection strain $(070 \mathrm{H})$ recovered from a patient with suppurative osteomyelites. Strains with SCCmec IV presented other band profiles and were grouped in another cluster with similarity of $80.8 \%$. The only SCCmec Ia strain was not be allocated to any cluster. None of the evaluated strains were grouped with circulating known clones (Figure 1).

\section{Analysis of the results}

One risk factor for MRSA acquisition identified in the present study by univariate analysis was previous use of ciprofloxacin [OR: 8.75 (1.59-48.29), $\mathrm{p}=0.04$ ]. In addition to ciprofloxacin use, multivariate analysis revealed that working in a healthcare environment [OR:17.5 $(1.22-250.36), p=0.04]$ was also associated with the acquisition of MRSA. However, the latter finding is questionable since only one patient infected with MRSA worked in a healthcare setting (Table 1).

\section{Discussion}

In a non-outbreak context of outpatients with skin and soft tissue infection, $S$. aureus was the most frequently isolated Staphylococcus species. The presence of $S$. aureus as the main agent of skin and soft tissue infections highlights the importance of identifying this pathogen. The high frequency of isolation of $S$. aureus is a common phenomenon in patients with infections such as cellulitis, impetigo, fu- 


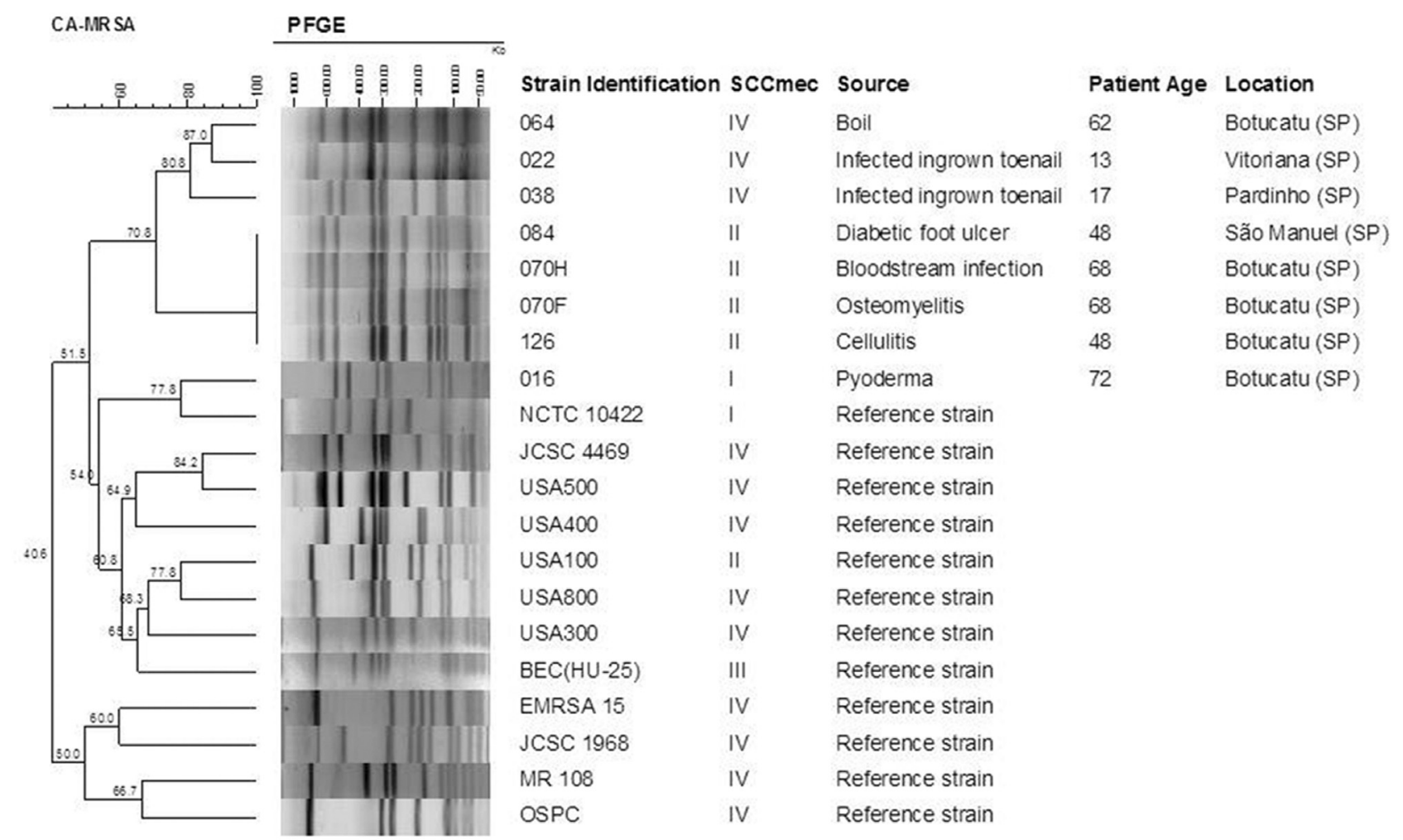

Figure 1 - PFGE analysis of MRSA isolates from skin and soft tissue infections. Three SCCmec II samples (070, 064 and 126) had identical profiles, while three SCCmec IV samples (064, 022 and 038) grouped with high similarity, and one sample (016) could not be allocated in any group.

runcles, carbuncles, and folliculitis (Stullberg et al., 2002), reaching a frequency of up to $72 \%$ (Issartel et al., 2002).

The PVL gene was detected in $15.1 \%$ of the S. aureus strains studied here. The presence of leukocidin in community-acquired strains has been extensively documented and is associated with methicillin resistance (Francis et al., 2005; Miller et al., 2005). However, in the present study PVL was not detected in methicillin-resistant strains, only in methicillin-sensitive strains. Demir et al. (2012) detected the PVL gene in $9 \%$ of $S$. aureus strains isolated from skin and soft tissue infections, but in other studies, the gene was absent in MRSA strains (Takizawa et al., 2005; Lee et al., 2010), supporting our findings.

None of the $S$. aureus or MRSA isolates carried the TSST-1 gene. The absence of PVL and TSST-1 gene in CA-MRSA strains isolated from skin and soft tissue infections was also verified in another study (Lee et al., 2010) suggesting involvement of other pathogenicity factors in such isolates.

The mecA gene was detected in seven strains isolated from skin infections of outpatients. SCCmec typing revealed the presence of types Ia, II and IV. A higher prevalence of types II and IV was also reported by Kikuta et al. (2011) in Japanese children with impetigo. In Brazil, detection of SCCmec type II was previously shown to be associated with hospital settings (Miranda et al., 2007; Schuenck et al., 2009). However, our findings indicate that patients who presented MRSA with this SCCmec type did not present an association with hospital environments nor with the healthcare worker included in our analysis.

The presence of such diversity types of SCCmec could be explained by the patient origin. They came from a community previously seen in an ambulatory care center, suggesting that this may constitute an intermediate zone. This zone may have the most variable types of SCCmec, characterizing by the prevalence and likely a coexistence of both CA- and HA-MRSA (Kouyos et al., 2013).

In a Brazilian study published in 2005, Ribeiro et al., described the first cases of CA-MRSA infections in Brazil. The MRSA isolates were collected from two patients presenting skin and soft tissue infections, and from another patient presenting septic arthritis. The three patients were from the community and had no risk factors for MRSA acquisition, such as the presence of medical devices, hospitalization or surgery in the last 12 months before MRSA isolation. Due to these characteristics, the origin of infection was considered community acquired. The samples were obtained between June 2002 and September 2003 in the outpatient clinic from two different hospitals in Porto Alegre, Rio Grande do Sul, Brasil. All samples harbored the SCCmec IV and presented identical profiles compared to the Ocean Southeast Pacific Clone (OSPC). In another study, Ribeiro and cols (2007) characterized CA-MRSA isolated in Rio de Janeiro and Porto Alegre. Besides the 
Table 1 - Statistical analysis ofdemographic data. Univariate and Multivariate analysis reveal the previous use of ciprofloxacin as a risk factor for CA-MRSA acquisition, and multivariate analysis showed that healthcare work was a risk factor. The risk factor in univariate analysis is highlighted in red and the values (number of cases, non-cases, OR and p values) are in bold. We highlighted in yellow the additional risk factors according to multivatiate analysis.

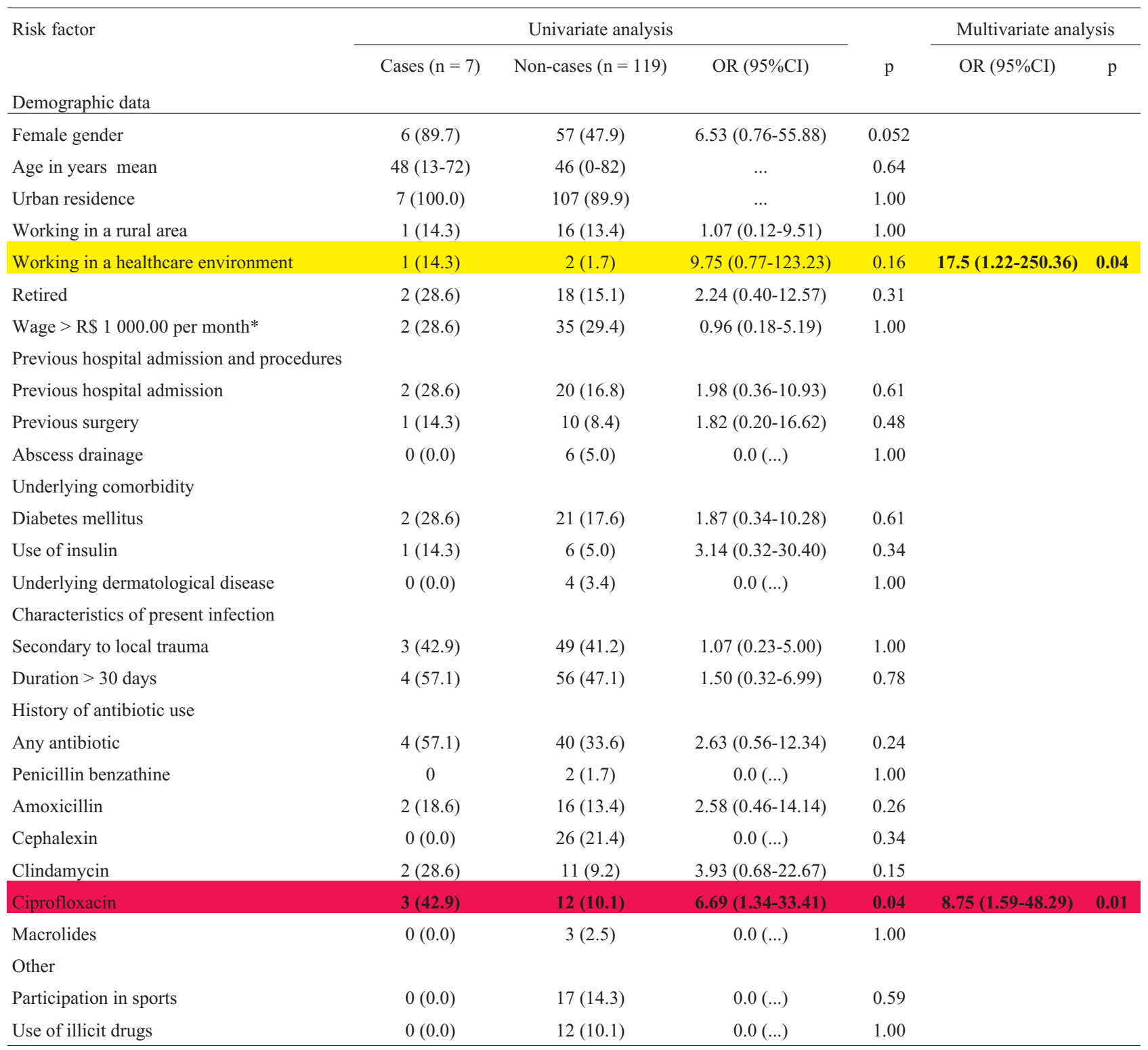

Results are numbers (percentage), unless otherwise stated.

*Approximately US\$ 500.00 per month.

$\mathrm{OR}$, odds ratio; $\mathrm{CI}$, confidence interval.

OSPC clone, they have found the USA300 and USA400 (Ribeiro et al., 2007). Other Brazilian studies verified that most samples carrying SCCmec IV presented identical profiles as the pediatric clone/USA800 (Miranda et al., 2007; Scribel et al., 2009; Carmo et al., 2011) and OSPC (Scribel et al., 2009) and strains highly associated with the USA400 clone (Schuenck et al., 2009). In Sao Paulo, Brazil, a new ST1176 strain emerged from a mutation in the yqiL locus, probably originating from the pediatric clone (Carmo et al., 2011). In our study the strains harboring the SCCmec IV were highly associated with each other, but lacked associa- tion with the most common clones by PFGE. In a study conducted in Europe, a large number of strains could not be related to previously known clones, a finding that might be explained by the wide diversity of CA-MRSA strains (Rolo et al., 2012).

The SCCmec II strains presented identical PFGE profiles. The strain SCCmec II isolated from one patient (patient number 070), that was isolated from osteomyelitis and bloodstream infection as well. It seems to be an invasive strain that deserves further characterization. The most con- 
cerning fact is its high similarity and presence in patients not related to each other.

Risk factor analysis revealed that patients carrying MRSA had a history of ciprofloxacin use and worked in a healthcare environment. The use of fluoroquinolones, particularly ciprofloxacin, has been shown to contribute to colonization with MRSA. S. aureus is highly resistant to fluoroquinolones while in sub-inhibitory concentrations can increase the adhesin expression (Bisognano et al., $2004,1997)$ thus leading to the success on MRSA colonization.

Healthcare workers play an important role in the dissemination of CA-MRSA in hospital settings and have been responsible for hospital outbreaks(Maltezou et al., 2009, Tang et al., 2007). Healthcare workers are prone to contracting community-associated resistant strains and represent an important source of infection with CA-MRSA for patients (Alia et al., 2012; Maltezou et al., 2009; Wagenlehner et al., 2008; Tang et al.; 2007; Raab et al., 2006). In our study, we verified that healthcare working was a risk factor for acquiring MRSA. However, this is a matter that needs to be validated by more specific studies and careful analysis in this regard.

In conclusion, we verified a low prevalence of MRSA in outpatients with skin infections, with strains not related with known MRSA clones, indicating a possible geographic clonal pattern. Risk factors indicate plausible routes of transmission since the use of ciprofloxacin and working in a healthcare environment are associated with the persistence of strains and dynamics of pathogen transmission. These findings have significant importance in CA-MRSA epidemiology.

\section{Conflict of interest}

The authors state no conflict of interest.

\section{Acknowledgments}

We thank São Paulo Research Foundation (FAPESP) (2009/09569-0) for the financial support. MFB was supported by a grant of CAPES. We thank Patricia Yoshida Faccioli-Martins and the medical residents who contributed substantially to the development of this study. Jim Hesson of AcademicEnglishSolutions.com proofread the English.

\section{References}

Alia H, Nasha JQ, Kearnsb AM, et al. (2012) Outbreak of a South West Pacific clone Panton-Valentine leucocidin-positive methicillin-resistant Staphylococcus aureus infection in a UK neonatal intensive care unit. J Hosp Infect 80:293-298.

Bisognano C, Vaudaux PE, Lew DP, et al. (1997) Increased expression of fibronectin-binding proteins by fluoroquinolone-resistant Staphylococcus aureusexposed to subinhibitory levels of ciprofloxacin. Antim Ag Chemot 41:906-913.
Bisognano C, Kelley WL, Estoppey T, et al. (2004) A RecALexA-dependent pathway mediates ciprofloxacin-induced fibronectin binding in Staphylococcus aureus.J BiolChem 279:9064-9071.

Boyle-Vavra S, Daum RS (2007) Community-acquired methicillin-resistant Staphylococcus aureus: the role of Panton-Valentine leukocidin. Lab Invest 87:3-9.

Campbell KM, Vaughn AF, Russell KL, et al. (2004) Risk factors for community-associated methicillin-resistant Staphylococcus aureusinfections in an outbreak of disease among military trainees in San Diego, California, in 2002. J Clin Microb 42:4050-4053.

Camargo CH, Cunha ML, Bonesso MF, Cunha FP, Barbosa AN, Fortaleza CM (2013) Systemic CA-MRSA infection following trauma during soccer match in inner Brazil: clinical and molecular characterization. Diagn Microbiol Infect Dis 76:372-4.

Carmo MS, Inoue F, Andrade SS, et al. (2011) New multilocus sequence typing of MRSA in São Paulo, Brazil. Braz J Med Biol Res 44:1013-7.

Chambers HF (1997) Methicillin resistance in staphylococci: molecular and biochemical basis and clinical implications. Clin Micr Rev 10:781-791.

Cooke FJ, Brown NM (2010) Community-associated methicillin-resistant Staphylococcus aureus infections. British Med Bull 94: 215-227.

Cunha MLRS, Peresi E, Calsolari RAO, et al. (2006) Detection of enterotoxins genes in coagulase-negative Staphylococci isolated from foods. Braz J Micr 37:64-69.

D'Agata EMC, Webb GF, Horn MA, et al. (2009) Modeling the Invasion of Community-Acquired Methicillin-Resistant Staphylococcus aureus into Hospitals. Clin Infect Dis 48:274-84.

David MZ, Rudolph KM, Hennessy TW, et al. (2008)Molecular epidemiology of methicillin-resistant Staphylococcus aureus, rural Southwestern Alaska.Em Infect Dis 14:16931699.

Demir T, Coplu N, Bayrak H, et al. (2012) Panton-Valentine leucocidin gene carriage among Staphylococcus aureus strains recovered from skin and soft tissue infections in Turkey. J AntimicrobChemother 67:837-840.

Francis JS, Doherty MC, Lopatin U, et al. (2005) Severe community-onset pneumonia in healthy adults caused by methicillin-resistant Staphylococcus aureus carrying the Panton-Valentine leukocidin genes. Clin Infect Dis 40:100-7.

Goering RV, Shawar RM, Scangarella NE, et al. (2008) Molecular epidemiology of methicillin-resistant and methicillinsusceptible Staphylococcus aureusisolates from global clinical trials. J Clin Microb 46:2842-2847.

Gosbell IB (2004) Methicillin-Resistant Staphylococcus aureus. Am J Clinic Dermatol 5: 239-259.

Issartel B, Tristan A, Lechevallier S, et al. (2005) Frequent carriage of Panton-Valentine leucocidin genes by Staphylococcus aureusisolates from surgically drained abscesses. J Clin Microb 43:3203-3207.

IWG-SCCmec. International Working Group on the Staphylococcal Cassette Chromosome elements. [home page on the Internet]. [place unknown: publisherunknown]; [updated unknown; cited June 7, 2011]. Available from: http://www.sccmec.org/Pages/SCC_TypesEN.html 
Jevons MP(1961) “Celbenin"-resistant staphylococci.British Med J 124-125.

Johnson WM, Tyler SD, Ewan EP, et al. (1991) Detection of genes for enterotoxins, exfoliative toxins, and toxic shock syndrome toxin 1 in Staphylococcus aureus by the polymerase chain reaction. J Clin Micr 29: 426-430.

Kikuta H, Shibata M, Nakata S, et al. (2011) Predominant dissemination of PVL-negative CC89 MRSA with SCCmectype II in children with impetigo in Japan. Int J Pediatr 2011: 1-8.

Kouyos R, Klein E, Grenfell B (2013) Hospital-Community Interactions Foster Coexistence between Methicillin-Resistant Strains of Staphylococcus aureus. PLoS Pathog 9:e1003134.

Kreienbuehl L, Charbonney E, Eggimann P (2011) Communityacquired necrotizing pneumonia due to methicillin-sensitive Staphylococcus aureus secreting Panton-Valentine leukocidin: a review of case reports. Ann Intensive Care 1:52.

Lee SS, Kim YJ, Chung DR, et al. (2010) Invasive infection caused by a community-associated methicillin-resistant Staphylococcus aureus strain not carrying Panton-Valentine leukocidin in South Korea. J Clin Microbiol 48:311-313.

Lina G, Piémont Y, Godail-Gamot F, et al. (1999) Involvement of Panton-Valentine leukocidin-producing Staphylococcus aureus in primary skin infections and pneumonia. Clin Infect Dis 29:1128-1132.

Maltezou HC, Vourli S, Katerelos P, et al. (2009) PantonValentine leukocidin-positive methicillin-resistant Staphylococcus aureus outbreak among healthcare workers in a long-term care facility. Int J Infect Dis 13:401-406.

Miller LG, Perdreau-Remington F, Rieg G, et al. (2005) Necrotizing fasciitis caused by community-associated methicillin-resistant Staphylococcus aureus in Los Angeles. N Engl J Med 352:1445-1453.

Miranda OP, Silva-Carvalho MC, Ribeiro A, et al. (2007) Emergence in Brazil of methicillin-resistant Staphylococcus aureus isolates carrying SCCmecIV that are related genetically to the USA800 clone. Clin Microbiol Infect 13:1165-1172.

Murakami K, Minamide K, Wada K, et al. (1991) Identification of methicillin-resistant strains of staphylococci by polymerase chain reaction. J Clin Microb 29: 2240-2244.

Milheiriço C, Oliveira DC, Lencastre H (2007) Update to the multiplex PCR strategy for assignment of mecelement types in Staphylococcus aureus. Antimicrob Ag Chemother 51:3374-3377.

McDougal LK, Steward CD, Killgore GE, et al. (2003) Pulsed-field gel electrophoresis typing of oxacillin-resistant Staphylococcus aureus isolates from the United States: establishing a national database. J Clin Microbiol 41:5113-5120.

Pereira VC, Martins A, Rugolo LMSS, et al. (2009)Detection of oxacillin resistance in Staphylococcus aureusisolated from the neonatal and pediatric units of a Brazilian Teaching Hospital. Clin Med Ped 3: 23-31.
Raab U, Kahlau D, Wagenlehner F, et al. (2006) Prevalence of and risk factors for carriage of Panton-Valentine leukocidin-positive methicillin-resistant Staphylococcus aureus among residents and staff of a German nursing home. Infect Control Hosp Epidemiol 27:208-211.

Ribeiro A, Dias C, Silva-Carvalho MC et al. (2005) First report of infection with community-acquired methicillin-resistant Staphylococcus aureus in South America. J Clin Microbiol 43:1985-1988.

Ribeiro A, Coronado AZ, Silva-Carvalho MC et al. (2007) Detection and characterization of international communityacquired infections by methicillin-resistant Staphylococcus aureus clones in Rio de Janeiro and Porto Alegre cities causing both community- and hospital-associated diseases. Diagn Microbiol Infect Dis 59:339-345.

Rolo J, Miragaia M, Turlej-Rogacka A, et al. (2012) High genetic diversity among community-associated Staphylococcus aureus in Europe: Results from a multicenter study. PLoS ONE 7:e34768.

Schuenck RP, Nouér SA, Winter CO, et al. (2009) Polyclonal presence of non-multiresistant methicillin-resistant Staphylococcus aureus isolates carrying SCCmec IV in health care-associated infections in a hospital in Rio de Janeiro, Brazil. Diagn Microbiol Infect Dis 64:434-441.

Scribel LV, Silva-Carvalho MC, Souza RR, et al. (2009) Clinical and molecular epidemiology of methicillin-resistant Staphylococcus aureus carrying SCCmecIV in a university hospital in Porto Alegre, Brazil. Diagn Microbiol Infect Dis 65:457461.

Stulberg DL, Penrod MA, Blatny RA (2002) Common bacterial skin infections.Amer Fam Phys 66:119-124.

Tang CT, Nguyen DT, Ngo TH, et al. (2007)An outbreak of severe infections with community-acquired MRSA carrying the Panton-Valentine leukocidin following vaccination. PLoS One2:e822.

Takizawa Y, Taneike I, Nakagawa S, et al. (2005) A PantonValentine leucocidin (PVL)-positive community-acquired methicillin-resistant Staphylococcus aureus (MRSA) strain, another such strain carrying a multiple-drug resistance plasmid, and other more-typical PVL-negative MRSA strains found in Japan. J Clin Microbiol 43:3356-3363.

Turlej A, Hryniewicz W, Empel J (2011) Staphylococcal cassette chromosome mec (SCCmec) classification and typing methods: an overview. Pol J Microb 60:95-103.

Wagenlehner FM, Naber KG, Bambl E, et al. (2007) Management of a large healthcare-associated outbreak of PantonValentine leucocidin-positive methicillin-resistant Staphylococcus aureus in Germany. J Hosp Infect 67:114-120.

Yao D, Yu F, Qin Z, et al. (2010)Molecular characterization of Staphylococcus aureus isolates causing skin and soft tissue infections (SSTIs). BMC Infect Dis 10:133.

All the content of the journal, except where otherwise noted, is licensed under a Creative Commons License CC BY-NC. 